\title{
The Perception of Workplace Incivility, Social Support, and Job Outcomes among Frontline Service Employees: A Cluster Analysis Approach
}

\author{
Boshra Hejraty Namin
}

Norwegian School of Hotel Management, University of Stavanger

\begin{abstract}
Workplace incivility is identified as a prevalent problem in the organization with a detrimental effect on individual and organizational outcomes. The aim of the current paper is to investigate the perception of workplace incivility and social support among frontline service employees. Moreover, this study explores how their demographic characteristics and working conditions are associated with their perception of workplace incivility and related job outcomes. A cross-sectional study was conducted, and the questionnaire was delivered to a sample of Norwegian frontline service employees. A total of 291 completed questionnaires (69.2\% response rate) were used for correlation analysis, one-way ANOVA, and cluster analysis. The results confirmed the connection between the perception of workplace incivility and job outcomes that has been identified by previous research. Cluster analysis revealed three distinct clusters of employees with interesting patterns and demonstrated that those who perceive higher levels of workplace incivility and lower levels of social support show higher negative job outcomes. It was also revealed that employees with longer tenure deal more effectively with workplace incivility and show better job outcomes.
\end{abstract}

Keywords: caring climate; coworker incivility; customer incivility; LMX quality; turnover intention 